This item was submitted to Loughborough's Research Repository by the author.

Items in Figshare are protected by copyright, with all rights reserved, unless otherwise indicated.

\title{
Supporting adolescent athletes' dual careers: The role of an athlete's social support network
}

PLEASE CITE THE PUBLISHED VERSION

https://doi.org/10.1016/j.psychsport.2018.06.007

\section{PUBLISHER}

(c) Crown. Published by Elsevier

\section{VERSION}

AM (Accepted Manuscript)

\section{PUBLISHER STATEMENT}

This paper was accepted for publication in the journal Psychology of Sport and Exercise and the definitive published version is available at https://doi.org/10.1016/j.psychsport.2018.06.007

\section{LICENCE}

CC BY-NC-ND 4.0

\section{REPOSITORY RECORD}

Knight, Camilla J., Chris Harwood, and Paul A. Sellars. 2019. "Supporting Adolescent Athletes' Dual Careers: The Role of an Athlete's Social Support Network”. figshare. https://hdl.handle.net/2134/34075. 
RUNNING HEAD: Dual-career of elite youth athletes

2

3

4

5

6 7

\section{6}

Camilla J. Knight ${ }^{1,2}$, Chris G. Harwood ${ }^{3}$ \& Paul Sellars ${ }^{1}$
${ }^{1}$ School of Sport and Exercise Sciences, Swansea University

Supporting adolescent athletes' dual careers: The role of an athlete's social support network

Acknowledgements: We gratefully acknowledge the funding received from the International Olympic Committee Advanced Research Programme to support this project.

7 Authors Note: Since the completion of this study, Paul Sellars has subsequently moved to

8 Cardiff School of Sport, Cardiff Metropolitan University.

9
This is the final draft of the manuscript accepted for publication in the Psychology of Sport and Exercise, and copyright is retained by the journal publishers. Once published the final draft of the manuscript will be available from https://www.journals.elsevier.com/psychology- of-sport-and-exercise 23 
27 Objectives: The objectives of this study were to, (a) understand the role of the social support network in facilitating a dual career in sport and education, and; (b) gain insights into the factors that may optimize the provision of such support.

Design: A two-stage qualitative study.

Method: In stage one, four different sport and education settings in the UK were examined: A tennis academy, a football academy, a national field hockey squad, and a high-performance swimming squad. Interviews were conducted with two athletes and associated significant others from each setting. In stage two, nine current or former international athletes from a variety of sports and countries recalled their experiences of managing a dual career. Analysis was conducted following the procedures outlined by Miles, Huberman, and Saldaña (2014).

Results: Overall, athletes in stage one and two perceived that to maintain their dual careers, they were heavily dependent upon the support of significant others. The role of the support network was to recognize the demands of a dual career, anticipate problems, value education, minimize barriers, and create autonomy-supportive environments. Key factors to optimize such support were focusing on the whole person, providing integrated support, and fostering a culture that promotes continuing education.

Conclusions: Results indicate that athletes require particular types of support within their home, at school, and in their sport context to be able to manage their dual career. However, a range of individual and group-level factors may influence the extent to which such support is available to athletes. 
Over the past decade, there has been a growth in research examining the dual careers

51 (i.e., combined sporting and vocational/schooling careers) of elite athletes (e.g., Debois,

52 Ledon, \& Wylleman, 2015; McCormack \& Walseth, 2013; Ryba, Stambulova, Ronkainen,

53 Bundgaard, \& Selänne, 2015). The stimuli for such research has been the recognition of the

54 individual and societal benefits that arise when athletes maintain their education or a

55 vocational career while competing on the international stage (cf., European Commission

56 2007, 2011). Research has identified that athletes who gain academic qualifications are better

57 prepared to manage transitions, cope with expected and unexpected exits from sport, and have

58 the potential to make a positive contribution to the workforce beyond sport (Torregrosa

59 Ramis, Pallarés, Azócar, \& Selva, 2015). Additionally, many athletes are unable to maintain a

60 living from their sport career earnings for an extended period of time (cf., McCormack \&

61 Walseth, 2013), so ensuring athletes can start a "second career" when they retire is necessary.

Athletes usually demonstrate high levels of motivation for the maintenance of a dual

career (Lupo et al., 2015). However, it can be a challenging proposition and one that is a

concern for most high-performance athletes (Ryba et al., 2015). Such challenges arise because

65

66

67

success in both elite sport and education/work require extensive time commitments (Ryan,

2015). Time commitments increase through childhood and particularly in adolescence as academic workload, along with training and competition load, increase rapidly (Borggrefe \& Cachay, 2012). Consequently, maintaining a dual-career can result in fatigue, a loss of motivation, a lack of opportunities to participate in activities outside of sport or education, as well as athlete overload and increased injury risk (e.g., McCormack \& Walseth, 2013).

Despite the potential negative consequences outlined above, the impact of engaging in a dual career on athletes' actual sporting success appears equivocal (Henry, 2010). There has been some indication that the simultaneous engagement in sport and academia could result in a decrease in sporting performance and has been reported as a concern for some coaches 
(Aquilina, 2013). However, there is also strong evidence demonstrating positive performance consequences (McCormack \& Walseth, 2013). For example, Aquilina examined the sporting and educational experiences of elite adult performers from France, Finland, and the UK. Athletes highlighted numerous benefits from their dual career including: the belief the skills learnt in one setting were transferred to others; the balance of intellectual and physical stimulation, which increased motivation and commitment to both domains, and; feelings of security in their sport, which allowed athletes to perform better.

Recognising the difficulties associated with maintaining education while participating in elite sport, the European Commission and the International Olympic Committee (IOC) have acknowledged the need to research and promote policies regarding athletes' engagement in education (cf. Amara, Aquilina, Henry, \& PMP, 2004; Aquilina \& Henry, 2010). Such attention to understanding and developing sport and education policies is important to increase opportunities for adolescent and young adult athletes to succeed in academic and sporting domains. However, such a focus on policy and organisational factors associated is insufficient if we fail to concurrently understand the more proximal support athletes require. Aquilina (2013) explored the social support network university student-athletes utilized to manage their university and sport involvement. At the more distal levels, Aquilina highlighted the role of the European Union and the International Olympic Committee, elite training centres, NGBs, the state, researchers, and medical staff in supporting student-athletes. At the most proximal level, the need for support from academic staff, clubs, peers, coaches, and family were highlighted. Such findings have been supported across many studies of athletes engaged in tertiary (i.e., university) education (e.g., Brown et al., 2015; Carless \& Douglas, 2009) and together provide extensive insights into the lives of university student-athletes. In contrast, our understanding of the support needed by talented adolescent athletes to maintain their dual career before they reach university has received scarce attention (cf. 
100 Christensen \& Sørensen, 2009). Some insights can be gained from recent studies examining

101 successful talent development environments (e.g., Henriksen, Stambulova, \& Roessler,

102 2010a, 2010b, 2011; Larsen, Alfermann, \& Henriksen, 2013), which have highlighted the

103 proximal support needs of prospective elite athletes. For instance, Larsen et al. (2013)

104 examined the environmental characteristics of a successful under-17 male soccer team. Most

105 pertinent for the current study, the players perceived that maintaining a dual career was

106 particularly demanding. Players indicated being largely dependent upon tangible and

107 informational support provided by their school, as well as regular communication between the

108 club, the school, and their parents to manage school and training demands. Clearly, given the

109 complexity associated with a dual career, specific examination of this area is warranted to

110 ensure that athletes receive optimal support.

111 Particularly, there is a specific need to understand what support is required for mid to

112 late-adolescent athletes because, from the ages of $15 / 16$ to 18 years, athletes encounter a

113 critical time of life as they experience multiple demands and transitions, many of which are

114 associated with athletes' engagement in dual careers (e.g., Stambulova, Engström, Franck,

115 Linnér, \& Lindal, 2015). As highlighted within the Holistic Athletic Career model, during

116 mid-late adolescence athletes may be transitioning (or considering transitioning) from

117 secondary to tertiary education, experiencing changes in their psychosocial support, and also

118 potential changes in financial support (Wylleman, Reints, \& De Knop, 2013). These changes

119 may co-exist with elevated athletic demands and thus, mid-to-late adolescence is a time when

120 support for a dual career is likely to be particularly important.

121 The need for such support has been illustrated in two recent studies of adolescent

122 athletes (Baron-Thiene \& Alfermann, 2015; Stambulova et al., 2014). For instance,

123 Stambulova and colleagues (2014) examined the social and cultural influences on the dual

124 career experiences of Swedish adolescent athletes at sport schools. Reinforcing the findings 
125 from university studies, participants indicated that they searched for and relied on external

126 support from their families, home coaches, and the staff at their school to manage the

127 demands they encountered. However, the specific types of support athletes' desired and the

128 ways in which such support was provided was not discussed. In fact, beyond knowing that

129 support is required, we are unaware of what support is required from individuals surrounding

130 athletes to enable them to successfully maintain a dual career.

131 If we are to create effective policies to facilitate athletes' dual careers, generate

132 guidelines for organizations or clubs, or provide education to coaches, parents, teachers or

133 athletes, we must generate clearer insights into the specific support needs for such adolescent

134 athletes. To this end, the objectives of this study were to, (a) understand the role of the social

135 support network in facilitating a dual career in sport and education, and; (b) gain insights into

136 factors that help optimize the provision of such support.

\section{Method}

\section{Methodology}

To address the aforementioned research questions, a two-stage qualitative design was

140 adopted. Such an approach was deemed appropriate for the current study as we sought to

141 understand the experiences athletes and members of their social support networks have of

142 providing and receiving support for a dual career. The study was positioned within the

143 interpretivist paradigm, underpinned by ontological relativism and epistemological

144 constructionism. That is, within this study, it was assumed that reality is multiple and

145 subjective and that knowledge is socially constructed (Sparkes \& Smith, 2014).

146 Stage one focused on understanding the support required from, and delivered by,

147 members of adolescent athletes' support network across a variety of sports within the UK.

148 The focus on various sports was driven by a recognition that opportunities to combine

149 education and training/competition vary across sports (Knight \& Holt, 2011), that the motives 
150

151

152

153

154

155

156

157

158

159

160

161

162

163

164

165

166

167

168

169

170

171

172

to maintain a dual career might be influenced by the potential to make a living from their sport (cf. Christensen \& Sørensen, 2009), and the end of compulsory schooling might be particularly challenging (Wylleman \& Lavallee, 2004). As such, stage one focused on athletes and members of their support network from four sports, which were selected based on: 1)

Education and sport combination (traditional schooling; traditional school and academy provision; sport academy and online/home schooling); 2) Sport type (individual, traditional Olympic sport; team, traditional Olympic sport; individual, professional sport; team, professional sport), and; 3) Athletes' age (athletes aged 15-16 years, completing their GCSE$^{1}$ exams and athletes aged 16-18 years, currently completing higher education awards). The aim of such criteria was to achieve maximal variance between participants.

Having completed stage one, stage two sought to examine the extent to which the findings from adolescent participants in the UK aligned with, or could be corroborated by, elite athletes in different sports and countries who had achieved success in their sport while maintaining their education. Specifically, stage two sought to examine the adolescent dualcareer experience of athletes who had achieved at the highest levels (e.g., competed at Olympic Games and World Championships) in their sport while also completing their school and/or university education (i.e., dual career attention). Such retrospective accounts were deemed valuable as they provided the perspective of athletes who had experience of succeeding in sport while studying, and as such were in a position to reflect on the long-term impact and value of the support they received as an adolescent athlete.

\section{The Research Team}

In line with an interpretivist paradigm, it is important to note the roles and experience of the research team. The first and second author were both responsible for developing the

\footnotetext{
${ }^{1}$ The General Certificate of Secondary Education (GCSE) is an academic qualification, generally taken in a number of subjects by pupils as their end their compulsory secondary education in England and Wales.
} 
173 research idea and questions. The first author was also responsible for conducting some of the

174 interviews in stage one, all of the interviews in stage two, and leading the data analysis and

175 writing. The second author acted as a critical friend throughout analysis and writing and the

176 third author conducted the majority of interviews in stage one.

177 When starting this study, the first author had approximately eight years of experience

178 as a qualitative researcher within youth sport, with a specific focus on the role of parents.

179 Further, she had approximately 15 years of experience of studying while competing in sport.

180 The second author had over 20 years of experience as a youth sport researcher, with a

181 particular focus on parents and coaches. He also had over 30 years of combining sport and

182 education/work. Finally, the third author was a postgraduate student completing his MSc by

183 Research and had experience of playing county and regional-level rugby union while

184 completing his compulsory and higher education.

185 Stage one

186 Participants and Sampling. Aligned with the desire to sample a range of sport and

187 schooling set-ups, four sporting set-ups were selected from which to sample participants: 1) A

188 tennis academy, in which athletes complete their education and sport at one venue; academic

189 work is provided through an online education system overseen by a tutor; 2) National field

190 hockey squad in which athletes attend a regular school, access training and competition

191 through local clubs and leagues, with monthly national training events and international

192 competitions, 3) A high performance swimming squad comprising international swimmers,

193 accessing education through the traditional school system and completing their training before

194 and after the school day; and, 4) A football academy which caters for elite football players

195 who complete their education through the traditional school system to the age of 16 years and

196 study at the football academy from the ages of 16-18 years.

197 Within each sporting set-up two athletes, one aged 15/16 years completing their 
198

199

200

201

202

203

204

205

compulsory education and one aged 17/18 years engaged in further education (e.g., 'A'

Levels or BTEC study²), who were deemed to be successfully managing their dual career

based on feedback from the head coach and/or manager were selected to participate. During

the athlete interviews, each athlete was asked to visually and verbally identify the members of their support network they perceived to be particularly influential in their dual career. Such athlete-centered choices were deemed important so as to avoid any external presumptions or predetermination of significant others. Having identified the people they deemed to be influential, approval to contact them was gained from the athletes and then consent to participate was gained from the individuals themselves. All but two individuals (one footballer's mother and one teacher) agreed to participate. Table 1 provides detail on the participants from each sport and Table 2 provides the overall demographic information.

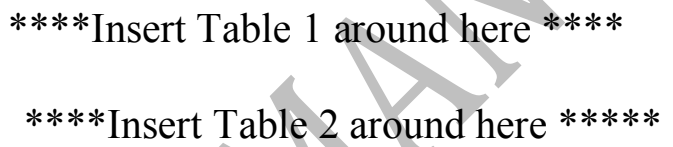

Procedure. On receipt of institutional ethical approval, appropriate venues from which to recruit participants were identified based upon the research team's knowledge of different sports and through conversations with sport scientist and performance lifestyle advisors.

Having identified appropriate venues, approval was sought from the head coach to approach athletes deemed to be succeeding at managing a dual career. The athletes were approached and provided with an information sheet. Once athletes had responded, an appropriate time and location for an interview was identified. Prior to interviews, informed consent was received from the athletes and, where necessary, their parents.

Data collection. Data collection occurred through semi-structured interviews. The

\footnotetext{
${ }^{2}$ AS and A levels are the traditional qualifications offered by schools and colleges for 16-19 year olds. They generally focus on academic subjects, although some are work-related. AS levels are usually taken as the first part of an A level course, although they can be taken as a stand-alone qualification. BTEC (Business and Technology Education council) are specialist work-related qualifications that combine practical learning with theory content. They are usually conducted between the ages of 16 and 19 years.
} 
220 interview guides for athletes and support network members were developed based on the

221 previous literature on dual careers, transitions, and social support in youth sport (e.g.,

222 Aquilina, 2013). The interviews started by asking the participant to describe their

223 involvement in sport/the athletes' sport and school. Next questions moved onto general

224 perceptions of dual careers and the benefits and challenges associated with maintaining school

225 and sport. The interviews then moved onto the main questions, asking the participants what

226 support they required or provided to enable the athlete to maintain their dual career.

227 Penultimate questions focused on factors that may impact upon the support required or

228 desired. Finally, the interviews ended with questions summarizing the key ideas identified

229 earlier in the interview. Interviews ranged in length from 30 to 87 minutes ( $M=47$ mins).

230 Data analysis. All interviews were transcribed verbatim and then read and re-read by

231 the first author to ensure immersion in the data. The transcripts from each participant from

232 each sport were read and each interview for each sport was then individually analyzed

233 following the qualitative data analysis procedures recommended by Miles, Huberman, and

234 Saldaña (2014). Analysis occurred in three stages. Firstly, descriptive codes (e.g., talking to

235 athlete, structuring communication, making time for others) were allocated to the data to

236 identify the raw data themes. Next interpretive codes, such as help timetabling and

237 understanding demands, were generated. These codes group descriptive codes into more

238 abstract concepts. Finally, pattern codes (e.g., understanding of the demands of a dual career,

239 valuing education) were identified, which demonstrated relationships between the interpretive

240 codes and provide an indication of the overarching ideas contained within each sport. A

241 summary of the overall findings for each sport was then constructed, prior to comparing the

242 codes across sports. Cross-sport analysis occurred by placing the pattern codes from each of

243 the sports within data matrices to allow for systematic review of the data.

244 Stage Two 
Participants. Nine elite (current or former) adult athletes from nine different countries

246 (Australia, Bahrain, Croatia, Iran, New Zealand, Slovakia, South Africa, Switzerland, and the

247 USA) participated in stage two. Two of the participants were still competing while the other

248 seven had retired from their sport. Participants competed in swimming, shooting, pentathlon,

249 sailing, alpine ski-racing, and badminton ${ }^{3}$, and all had attended at least one Olympic games or

250 World Championships as well as European, All-African, and Commonwealth games. The

251 participants had attended 12 Olympic Games and 24 World-Championships, amassing a total

252 of seven gold medals, 13 silver medals, and five bronze medals across these championships.

All of the participants had completed their compulsory (secondary) education while

254 competing, in one of three varieties of institutions: Four participants had attended a private

255 school, two attended a sport school, and three had completed their schooling through the

256 mainstream system in their country. While competing, six had also obtained a Bachelors

257 degree, and two a Masters degree. Of the seven retired athletes, one was in full-time education

258 and the other six were in full-time employment. Since retiring, two participants had

259 completed a bachelor degree, one obtained a Masters degree, and one completed a PhD.

260 Procedure. Following the completion of phase one, phase two was carried out.

261 Through communication with the International Olympic Committee, potential participants

262 who had maintained a dual career while competing, achieved at the highest level in their

263 sport, and were able to converse in English were identified. These individuals were sent an

264 email outlining the study purpose and procedures, along with a consent form. Participants

265 who were interested in taking part in the study then contacted the lead author who organized a

266 suitable time for a Skype interview.

267 Data collection. Data were collected through semi-structured interviews. The interviews

268 began with a brief overview of the purpose of the study as well as a review of important

\footnotetext{
${ }^{3}$ In the results, Participant $1=$ Sailor; Participant 2,5, 7, and $8=$ Swimmers; Participant $3=$ Shooting; Participant 4 = Heptathlon; Participant $6=$ Badminton; Participant $9=$ Skiing,
} 
ethical considerations such as confidentiality and completion of the consent form. Participants were then asked to provide an overview of their training and school schedule throughout their adolescence and early adulthood, providing insights into their overall experiences of a dual career during this time. The majority of the interview then focused on how different members of their support network facilitated or detracted from their adolescent dual career and the identification of suggestions to enhance the support significant others provide to athletes. Next participants were given a summary of the findings from stage one and asked to provide any insights into the similarities or differences with their experiences. Interviews ended with a review of the main areas covered and an opportunity for athletes to give further information. On completion of the interview participants were sent a copy of the recommendations from stage one for comment. Interviews ranged in length from 72 to 126 minutes ( $M=88$ mins). Data analysis. Stage two data collection and analysis began following the completion of the analysis of stage one data. The data were analysed using the same process of identifying descriptive codes, interpretive codes, and pattern codes as adopted in stage one. The findings from stage two were then compared to those from stage one to enable the identification of similarities and differences between experiences. This facilitated the identification of any factors that may impact upon the support provided or required for adolescent athletes to maintain a dual career.

\section{Methodological Rigor}

It has been suggested that a relativist rather than criterion approach to judging the quality of qualitative research is appropriate (Sparkes \& Smith, 2009). Aligned with this approach, we have drawn upon the nine suggestions of Smith and Caddick (2012) that we deemed appropriate for the current study. However, we recognize that these criteria are openended and subject to reinterpretation and encourage the reader to make their own judgments. 
Specifically, we sought to produce a paper that provided a substantive contribution to

294 the understanding of dual careers from the perspectives of athletes and members of their

295 social support network. The justification for this is provided through the manuscript

296 introduction. We perceive that through the use of extensive interviews in a novel area that this

297 study will have impact by encouraging the generation of new research questions, as well as

298 encouraging those working with athletes to seek to develop strategies to enhance the support

299 they provide to young athletes engaged in a dual career. Through the use of pilot interviews, a

300 two-stage study process, as well as the involvement of athletes and members of their social

301 support network across a range of sporting and education set-ups, and the provision of

302 numerous quotes within the results we have sought to demonstrate width or

303 comprehensiveness of evidence. Further, we have attempted to demonstrate coherence by

304 ensuring continuity between the philosophical underpinnings of the study, the research

305 question, the data collection and analysis methods, and the presentation of the results.

306 Catalytic and tactical authenticity is exhibited through the interest the findings of this

307 study have garnered from the sports organizations involved and their desire to make change

308 based upon subsequently developed recommendations. By encouraging participants to select

309 the individuals they deemed most influential in their dual career and asking participants in

310 stage two to reflect on the findings produced in stage one, we sought to remove notions of the

311 research team as the experts. Rather, we critiqued this approach and sought to empower the

312 participants to demonstrate their expertise. Through detailed descriptions of the participants

313 involved in the study, the use of rich description of findings and extensive quotes, and also

314 the location of the current findings within previous literature, we hope the results resonate

315 with readers. Finally, credibility and transparency were sought through pilot interviews,

316 engagement with multiple individuals within each sport in stage one and prolonged, detailed 
317 interviews in stage two, and spending multiple days in different sport set ups in stage one to 318 gain broader insights beyond the interview data.

\section{Results}

In stage one and two, the athletes discussed a range of benefits associated with their

321 dual-career, particularly the value of having education to "fall back on" if their sporting career

322 was unsuccessful. Despite the benefits, participants also highlighted a number of challenges.

323 Support from members of their support network was seen to be particularly important in

324 enabling the maintenance of school and elite sport. Overall, participants from stage one and

325 two generally indicated that they were reliant upon support from their parents (particularly

326 their fathers within football), their coach, teachers/tutors, and where available performance

327 lifestyle or welfare advisors. Certain individuals mentioned peers or siblings in passing but

328 compared to others, their support appeared to be less influential.

Although there were differences in the experiences of the participants in stage one and

330 two, there was much similarity in the roles of the support network in helping them maintain

331 their dual career as an adolescent. As such, in the following sections, data from stage one and

332 two are combined ${ }^{4}$. First, the characteristics that comprise the role of the dual career support

333 network are detailed. Secondly, fâctors that enable support to be optimized are described.

\section{Role of the Support Network}

335 The role of the support network appeared to be characterized by the five themes.

336 Namely, the support network: (a) Recognize dual career athletes' needs and make adaptive

337 changes, (b) Anticipate barriers and suggest solutions, (c) Demonstrate a belief in the value of

338 education and a dual career, (d) Remove barriers to maintenance of a dual career, and (e)

339 Create an autonomy-supportive environment to foster dual career athletes' independence.

\footnotetext{
${ }^{4}$ To allow for distinction between participants from stage one and two, pseudonyms have been given to the stage one participants, and numbers have been given to stage two participants.
} 
341 highlighted the need for members in the support network to recognize the demands of

342 engaging in a dual career and change their support in response. This may include recognizing

343 the day-to-day demands athletes' experience and altering expectations or requirements as

344 demonstrated by the father of one of the swimmers, "She [Cally] gets tired, sometimes after a

345 hectic week training...Y You can see it and maybe that will affect her concentration a bit. Rude.

346 Obviously, we'll sort of pinpoint that, we understand." Similarly, a performance lifestyle

347 advisor for the tennis players shared her understanding of demands, explaining, "I don't envy

348 them. A 15-year-old trying to do his homework at school every day finds it tough enough, let 349 alone when you're travelling and you're having to try and study in between matches."

350 By recognizing the challenges of the dual career, the support network could adapt their

351 behaviors to help athletes in positive manners. Numerous examples of changes in behaviors in 352 response to the demands were provided, ranging from reducing the number of chores at home, 353 to providing flexible school timetables, and changing training timetables to facilitate school 354 commitments. As Hannah's swim coach said, "I can say, 'well actually if you're stressed 355 today perhaps you don't need to do that type of training, perhaps do a different type of 356 training." Similarly, the mother of a swimmer (Lisa) explained, "I think as a parent you cut 357 her that slack almost because of the hours that she's doing in there." In contrast, Participant 8 , 358 who was also a swimmer but had a more difficulty maintaining her dual-career, described the 359 challenges she encountered because her school was not flexible, "I was away for 3 months 360 and when I got back there was no support in me catching up on school work ... the principal 361 called up my parents and said to them they weren't willing to help me catch up." If the demands athletes encountered were not recognized or adapted to, the participants 363 recalled many more issues with the maintenance of a dual career. However, by changing their 364 behaviors to meet athletes' needs, members of the support network displayed an appreciation 
and recognition of what athletes were trying to achieve, which appeared to help athletes maintain their motivation and enjoyment of their dual career. As Hannah summarized in relation to understanding from her school, "My head teacher was the best, ...he'd support swimming and school at the same time; he'd always make sure that I was ok in school."

Anticipate problems and suggest solutions. In addition to recognizing the general demands of a dual career, responding to specific challenges that arise at certain times or in certain situations was also deemed necessary. For example, Dillon's (one of the tennis players) strength and conditioning coach explained how they changed their program for athletes who got "edgy" and "anxious" around exam time. As he said, "we're normally quite bespoke with their program about that time of year." Similarly, Brody explained how his football club anticipated challenges during exam times and changed their timetables:

I used to go out of school on Tuesday afternoon and Thursday afternoon, during the exam times obviously there is a lot more revision that you need to get done and deadlines that you need to meet, so the club is alright with me not training on Tuesday and Thursday afternoons and just come in on the evenings to get the work done.

The flexibility and understanding demonstrated by the school and the academy ensured that athletes felt capable of maintaining their dual career and maximised opportunities for success. Furthermore, the support network perceived that they should anticipate and plan for problems that may arise in specific situations. For example, the tennis players regularly travelled abroad and could encounter challenges if they did not have access to the Internet to complete their work. Recognizing this problem, Dillon's parents prepared strategies to limit any negative consequences. As Dillon's mother said, "Sometimes poor Internet [is an issue]... So even with that what we have to try and prepare for that by making hard copies of things that he's going to work on, so that he can at least sit down and work." Similarly, the 
mother of one of the tennis player's (Niamh), explained that she must prepare for unexpected issues such as sudden trips abroad. In those instances, she said it is all about:

You know, organizing the schedule with [coach] and making sure everything's up to date with that, if she needs any extra kind of things, extra schoolwork or extra physio and things like that, all of those things, all the things that cause panic.

The swimmer's teacher reiterated the importance of anticipating problems when travelling and helping athletes to proactively plan for them, explaining:

I think we've been quite active in making Lisa realise as well that she needs to be keeping the staff onside if you know what I mean, regarding making sure that she keeps them up to speed on when she's not going to be around and any work is caught up.

By anticipating potential problems and proactively providing or suggesting solutions, athletes were supported to manage the pressures associated with their two careers.

\section{Demonstrate a belief in the value of education and the benefits of a dual career.}

Athletes' support network provided a consistent demonstration of the importance of education and the value of maintaining a dual career. For example, Dillon's father said, "I think it's [education] really important and I have done from the beginning and the reason is I sum it up by saying 'nobody likes a sick ex-tennis player."' Similarly, the mother of a hockey player (Sofia) suggested, "A good education will set you in good stead...it's just something as a family that we, we feel strong about." The manner in which this belief was demonstrated varied across participants, with some placing slightly more emphasis on education than others. For instance, in tennis and football, members of the support team indicated that education was important, but it could be returned to at a later date; whereas in swimming and field hockey (traditionally associated with less financial reward) education was viewed as a "non-negotiable". Nevertheless, the consistent belief across the support team that continuing with education was beneficial seemed to be particularly important in helping athletes maintain 
their motivation to achieve in both domains. As Participant 6, a badminton player, explained: I just feel that I was so lucky that I really had the perfect combination for this [maintaining school and sport]...I think it can be easy for the teacher to say education is the first thing you do, and it can also be easy for the coach to say that sport should be your first thing you do. I think neither of those are true... all sides they just need to make sure that the kids stay on track on both.

Furthermore, the support team and the athlete valuing education seemed particularly

helpful because it prevented athletes from feeling conflicted. As Cally (a hockey player) said, "Yeah, I think its [valuing education] really important because I want to go to university and stuff, I want to be in physiotherapy and they [my support network] know and support that." Further, with everyone on the same page it helped to ensure that everyone's expectations were the same, which eased tension within the support network. This appeared to be particularly helpful for parents as a performance lifestyle advisor for tennis said, "I think if you have coaches that encourage it [education] then that gives the parents a bit of relief in knowing that the coach will support them as well."

In some of the sports, the value on education was demonstrated through specific support behaviors, such as members of the support team monitoring athletes' engagement in their academic work and holding athletes accountable for their attainment in this field. For example, explaining what helped with her education Niamh (a tennis player) explained that if she fell behind with her studies, her coach and tutor would say, "you have got to miss a couple of [training] sessions to get myself back where I needed to be.” The players viewed such monitoring positively because it kept them on track. As a footballer, Brody, said: Before the season starts, the club will talk to people at school to make sure I do have their permission to leave but also they have stopped people leaving school if they are falling behind, so that also shows how important they think education is, it's good. 
Minimize barriers to maintaining a dual-career. One of the fundamental

440

441

442

443 requirements to enable engagement in a dual career seemed to be having the necessary opportunities to complete their education and training, with minimal barriers. Quite simply, as Participant 3 said in relation to his shooting and school career, members of the support network must, "Make everything very convenient for the athlete I think." For such opportunities to arise, athletes indicated being dependent upon their education establishments to facilitate engagement in sport and sporting communities to support and enable academic engagement. Support from parents was perceived to play a critical role, as Harry's dad shared: There has been a lot of sacrifice involved and a lot of travelling up and down...but I said to myself I am not going to let my boys not have what I didn't have [i.e., opportunities to excel in sport], at least I can give them support.

Alongside the support for sport, significant others also provided support to succeed at school. Such support was apparent through flexible timetabling at school to facilitate football training, as Brody's head of year explained, "So what we did is we went through his timetable, we identified slots on a weekly basis where I can move him from his lessons." Additionally, parents or the Sports Federation facilitating access to tutors was seen as one of the most beneficial types of support for athletes in both study phases. Tutors were valued for various reasons, as a swimmer, Participant 5, stated, "I think the tutoring helped a lot of swimmers at the time because when they were swimming without them they were not able to focus on education." Athletes who spent extended time away from school for training and competitions (e.g., tennis players, sailors) particularly valued tutors.

On returning home from trips away, assistance from schools and parents was deemed to be extremely important in helping athletes to catch up on work. For example, Participant 9 shared her experience of returning from skiing trips saying: 
kid with a school to go to and papers to turn in and so the support to have things organized and not waste the week trying to collect all the bits and pieces that you've missed was definitely a massive, massive help.

467 Overall, the removal of barriers and presentation of opportunities appeared to be largely dependent upon a collective effort across the support network, with clear roles and responsibilities and regular communication across sport and education domains (discussed in 470 further detail under the factors section).

Create an autonomy-supportive environment to foster independence. Finally, there appeared to be a belief across the sports that athletes needed to have control over their education and sport decisions. Participant 3, the shooter, shared:

It was all about my responsibility I think the personal approach is the key because then you can face someone and you can speak on your behalf and then it's much more valued than if you send someone else to do that for you, so my mum she was helping with the administration let's say she helped kind of sending letters or whatever, but the most important stuff it was on my shoulders.

Similarly, one of the swimmers, Participant 7 said, "When I got to the age where I would go and talk to the teachers then they kind of respected that and they helped me do what I needed to do to prepare for being away for two weeks, or whatever it was."

483 to guide their own development, while in other sports it was through the active development 484 of independence in athletes. For example, Niamh explained, "I wanted to try and focus more 485 on the kind of the tennis side, that was kind of my decision this year, so I decided to stay and play and see how it goes really.” Dillon's mother expressed a similar view in relation to moving Dillon to the tennis academy, "I would ask him and he'd say, 'No I don't want to go to an academy full time yet,' so we waited ...I think we've always let Dillon lead the way." 
Athletes were also allowed to take control of their decisions regarding schoolwork. For instance, the hockey players had autonomy over their school work, as Sofia's teacher said, "If

491 she needs it, it's not that its being provided as such, but she knows that we are here and 492 available and she can use us if and when she needs to." By trusting athletes to make the 493 decisions that worked for them, the support network not only ensured the players felt in 494 control of their choices but also that they would be happy with the choices. In relation to her 495 swimming, Lisa explained:

Well you've taken your information and now it's down to you and if you want to do it then you do it and if you don't do it then don't moan when you don't do well. Like I think that's the sort of approach she [mother] has sometimes. Which is, I think that sort of makes me do it even more, knowing that they like trust me to do it.

500 However, although athletes were trusted to make their own decisions, the guidance of their support network helped athletes make informed decisions. As Hannah's father explained in relation to her swimming and school:

I leave the choices to them tell you the truth, because I'll say it's your choice, but I'll spell out the consequence that if you make this choice then this is what'll most probably happen, but I'll leave it ultimately down to them.

\section{Key Factors for Optimizing Support}

To enable significant others to provide the aforementioned support, it appeared that

508 three factors were important. Namely, participants indicated that, (a) focusing on the whole

509 person, (b) integrating efforts within the support network, and (c) fostering a culture that

510 promotes continuing education, would enable optimal support from their network.

511 Focus on the whole person. First participants reflected on the importance of the

512 support network focusing upon developing a whole person, rather than their sport or

513 schooling career individually. As a swimmer, Participant 7, explained in relation to coaches: 
My biggest recommendation to coaches is to look at your athlete as a person, as a whole and not just an athlete... When a coach is able to make that athlete a better person and a whole person, they haven't just succeeded at school or being a good person, they've succeeded as being a better athlete because they understand things better.

Another swimmer, Participant 8, argued for valuing all dimensions of an athlete, explaining, "I think that we need to be looking at the athlete as you know emotionally, socially, mentally ...you know the five dimensions." Such views were reiterated by a tennis coach, who shared, "the advice is that you develop not just a tennis player, but a whole person and surprisingly if you do that it actually adds to the other areas." By adopting such an approach, pressure on athletes' sporting or education careers were reduced as they remained in perspective.

Participants perceived that the focus upon the whole person and a dual career needed to extend to Sports Federations. For instance, based on a lack of support from her Federation Participant 1, a sailor, explained:

[Federations] should be saying, "we don't force anyone to do studies, but if there are students who feel the need [to study] for the future or their career, we will provide some tools and some support". But they don't and that for me was the most frustrating.

Overall, summing up the views of many, Participant 8 explained why federations should focus on the whole person, stating, "I think that the organizations have a duty of care to make sure that their athletes are going to be okay once they leave the squad."

Integrated efforts within the support network. To optimize support, and particularly to remove barriers to a dual career and increase a belief in education, individuals in the support network needed to work together rather than in isolation. As Dillon's tennis coach explained in relation to being on the same page as Dillon's tutor:

I need to know where he's at, what progress he's making and a little bit about, what is he being asked to do at the moment, what is the workload like, what should he be doing 

and particularly, if I go away with him then I'm going to need to know roughly what his targets are and what's expected of him when he's away.

The tutor reiterated the need for joined up thinking, stating, "We try and encourage them [players] to do work when they're away and if I know the coach who is away with them, making sure that they know that they should be doing work and stuff." Such an integrated approach was possible because individuals engaged in frequent and open communication. Participants also discussed the importance of parents and athletes working together. As the badminton player, Participant 6, explained:

The biggest support they [parents] gave to me was that you always had a very awkward conversation, they sat down with me, they tried to understand where I was coming from, and they always tried to work out solutions that works for both...we tried to find a solution, and that's the best support of all.

In addition, participants valued parents and coaches providing similar messages. For instance, a swimmer, Participant 7, said, "I think that [support for education] can also come from coaches, not just parents, because some athletes will know sometimes it's better coming from someone else than mum and dad you know, you listen to it a bit better." Summarizing the importance of an integrated approach, a sport psychologist explained:

Athletes can be feeling the pressure from everyone, making sure she has the right balance but nobody communicating with each other; they are all just telling her what the expectations are from their angle rather than sitting down and working out the best thing for that individual. I think that's the toughest one with sport and education.

Foster a culture of expectation around continuing education. Finally, participants emphasized the importance/benefit of members of the support network fostering a culture in which continuing education is an expectation - something that is not questioned. For many of the athletes, an expectation of continuing education was created by parents, as Participant 3 (a 
564 shooter), shared, "My parents are educated, both university education, so from beginning of

565 my sport career it was always depending on my school results so I was really motivated by

566 my parents to study well." Participant 7, one of the swimmers, extended this point, stating, "It

567 was never a question for my family of whether I was going [to school and university], ...it

568 was just an assumption....So I think that helped - the not questioning it."

569 When or if such a culture extended to the sport or society, it appeared to be particularly

570 helpful in supporting athletes' conversations with coaches, as Participant 6 said about his

571 badminton coach, "I didn't have to really negotiate my education [with my coach] because of

572 the culture and mentality of my country." In contrast, one of the tennis coaches reiterated the

573 importance of a cultural change in tennis in the UK, explaining, "it's about developing a

574 different culture in this country; people are out of school a lot and the majority of them

575 shouldn't be because they're never going to make it as tennis players anyway."

Such national expectations towards continuing education were not always without

challenges though, as Participant 5 explained:

I went to the Olympics and then after coming back there was just some discussion with the association that you know I was going to University next year, so he's not going to

Additionally, Participant 7 explained that sport also needs to be valued: way more important than sport. So that's a cultural mindset that we have to try and say, “okay we understand and we get that, and we're not saying that education isn't important but the two and two could work together."

587 Overall participants perceived that sport and social culture towards education influenced the extent to which they were able to obtain support necessary to maintain their dual career. 


\section{Discussion}

The objectives of this study were to, (a) understand the role of the social support

591 network in facilitating a dual career in sport and education, and; (b) gain insights into factors

592 that may optimize the provision of such support. The key intention was to engage in a more

593 rigorous understanding of the characteristics of support provided by the "team around the

594 athlete' by investigating the experiences of dual career management from the perspectives of

595 athletes and their social support network. Such viewpoints not only included current, high

596 performing adolescent athletes and their identified support team, but also retired elite,

597 international athletes who had pursued a dual career pathway.

\section{Roles of the Support Network}

One of the most striking elements to emerge from the results was the level of understanding needed from the support team with respect to the explicit demands of elite sport, education, and the interchange between the two achievement domains. To provide effective support parents, teachers, coaches, and organizational support staff demonstrated high levels of 'contextual intelligence' (Brown, Gould, \& Foster, 2005) and a cognitive

604 awareness of the stressors faced by the elite student-athlete (cf. Brown et al., 2015; Debois et 605 al., 2014). When members of the network lacked such contextual awareness, athletes and others experienced more challenges in their attempts to engage in a dual career or to support

607 such attempts. Such contextual intelligence appeared to be most apparent in settings where

608 there was a cultural (both within society and sport) acceptance of dual careers (e.g.,

609 swimming in the UK) likely due to the greater experience of the support team in this domain.

610 The results further highlight the need for perceived social support to be present in all its

611 multidimensional forms (cf. Rees \& Hardy, 2000). For example, parents and teachers

612 appeared to possess a consistent degree of readiness to offer tangible and logistical support

613 with a clear emphasis on being solution-focused and an adaptable, proactive ability to plan in 
614 ways that would remove unnecessary barriers for the athlete. Emotional support from parents

615 often took the subtle form of 'reading their child' and showing empathy in the context of the

616 simultaneous challenges (Harwood \& Knight, 2015). Such efforts were evident across the

617 idiosyncratic demands linked to specific sport types (e.g., international travel-school conflict

618 in tennis versus daily training schedule-school conflict in swimming) and showcased the

619 cognitive skills of network members in terms of anticipation, planning and adaptation.

620 Insights into the types of facilitative environment created by the support network

621 resonated with the principles of basic needs theory (Ryan \& Deci, 2002) in that athletes were

622 presented with autonomy-supportive opportunities to engage in developmental tasks and

623 organizational activities independently. In addition, care to ensure that athletes felt like they

624 could own their own journey and decisions about sport involvement were afforded by parents

625 (Knight \& Holt, 2014). Underpinned by collective contributions from all members of the

626 support network, such an autonomy-supportive environment seems to offer a sense of how

627 athletes may be able to optimize their self-regulation as individuals managing the integrated

628 transitions between sport participation and schoolwork/education.

629 Factors that Optimize Support Provision

630 Through the integration of interviews from individuals involved in a variety of sports, in

631 various education and sport set-ups, and across a range of countries, we sought to identify key

632 factors that may influence or optimize the provision of support. Somewhat surprisingly, the

633 experiences and needs of individuals in different countries and different sport/education set-

$\$ 34$ ups appeared to be much more similar than they were different. Nevertheless ${ }_{2}$ the overall data

635 set provide some intriguing insights into individual- and group-level factors that underscore

636 optimal support for aspiring athletes in a dual career reality. Importantly, when providing

637 effective support, the aforementioned support network attributes did not appear to exist in

638 isolation. Rather, these attributes existed across members who collaborated in manners that 
639

640

641

642

643

644

suggested a sense of seamlessness in terms of supporting the development of the whole person. Congruence between the support team extended beyond support to the athlete to broader group-level support as all network members supported each other. Such support enabled the entire network to be 'on message' with respect to supporting each other's decisions, subsequently reducing any confusion the athlete might experience from mixed messages or differential value judgements. Such a finding is consistent with Henriksen and colleagues (2010a), who have highlighted that integrated efforts are a critical characteristic of successful athletic talent development environments (ATDEs). Resonating with Martindale, Collins, and Daubney's (2005) reinforcement of coherent philosophies within a TDE, these findings accentuate the importance of sports federations facilitating stronger integration among different members of athletes' support network (cf., Harwood \& Knight, 2015). Notable features of the support network exalted by athletes and reinforced by members was the implicit belief in the dual values of sport and education (cf. European Commission, 2011). Congruent beliefs in the pursuit of holistic athlete development and a grounded, multidimensional identity (cf. Brewer, Petitpas, \& Van Raalte, 2017) appeared to promote behaviors in the support team that enabled the athlete to optimize engagement and development in both domains. A perceptive observation by one retired elite athlete also reflected how sociocultural expectations of the athlete valuing and continuing education were not always 'a given' or assumed in certain countries; yet such societal and organizational expectations (e.g., duty of care) were powerful and necessary in facilitating holistic development and healthier personal identities (European Commission, 2011). Further, the emphasis placed on sport versus education differed across some countries, sports organizations/types of sports, and within families. In situations where the country or organization placed less emphasis on education, family expectations around continuing education were of even greater importance. 
Overall, consistent with Henriksen and colleagues (2010a, 2010b) holistic ecological approach to talent development, and the suggestions of Aquilina (2013) and Sum et al. (2017) regarding influences on dual careers, the current study indicates that adolescent athletes'

667 successful engagement in a dual career appears to be influenced by interactions at and across

668 varying environmental levels. In drawing together our findings alongside pre-existing

669 literature, we propose that an athlete's dual career experience is directly influenced by a range 670 of factors including the contextual intelligence, values, and beliefs of individual members of a 671 support team located within the microsystem (Bronfenbrenner, 2005; Henriksen, 2010a, b).

672 Such contextual intelligence and behaviors of individuals appear to be individual

673 representations of the shared expectations and understanding of dual careers that the support

674 network possess. These representations may be reciprocally formed and informed through

675 consistent communication and interaction within the support network. Further still, such 676 values, expectations, and understanding may be more broadly influenced by the macro-level 677 culture (Henriksen, 2010a) created by individual national governing bodies and/or the societal 678 expectations that exist regarding engagement in education versus achieving success in sport.

\section{Limitations and Future Directions}

The current study provides important initial insights into how members of the support

681 team can support elite adolescent athletes as they attempt to complete their dual career.

682 However, such findings must be considered in light of the limitations of this study. Most

683 notably, the lack of negative examples included in the study and the limited experiences from

684 elite team sport athletes to guide the formulation of the findings. Moreover, the objective

685 educational success of the dual-career of the athletes were not explicitly measured (e.g., 686 academic grades, school achievements) in stage one as the participants were in the middle of 687 completing their courses. For the purpose of this study, we defined successfully pursuing a 688 dual career pathway as achieving on the national or international stage in sport while 
maintaining or completing compulsory education with necessary grades ${ }^{5}$ to facilitate further

691 between countries and cultures so further examination of what a "successful" dual career

692 actually is, whether at an individual, national, or international level, would be beneficial.

693 Finally, the views of individuals from educational institutions were more limited within this

694 study due to the challenges of engaging with them. As such there is a greater emphasis on the

695 views of individuals from within the sporting context than the educational context within the

696 findings. To extend the findings of this study, longitudinal studies incorporating the views of

697 all members of the support network would be beneficial to illustrate how support must change

698 and adapt across transitions and challenging points in the academic/sporting calendar. Further,

699 studies with athletes who were not able to complete a dual-career would help to more clearly

700 articulate the nature and consequences of support, or lack thereof.

\section{Conclusion}

The findings of this study provide a clear indication of the breadth and depth of

703 support required by adolescent athletes to maintain a dual career. Most importantly, it appears

704 that in addition to providing support to athletes, members within the athletes' support network must also support each other and share similar beliefs and expectations regarding sport and education. Such shared expectations and beliefs are more likely to be possible if a culture in

707 which continuing education is accepted and supported within sport is fostered, but this is

708 likely to be influenced by societal values towards sport and education. Overall, it appears that

709 identification of specific roles and responsibilities to be fulfilled by each member of a support

710 network, as well as an identification of the sporting and educational demands and goals of

711 athletes would be useful to guide support for a dual career during adolescence.

\footnotetext{
${ }^{5}$ In the UK sample, this was classed as a minimum of $\mathrm{C}$ grades in English, Maths, and Science at GCSE level as this is usually required as a minimum to engage in further and subsequently higher education.
} 


\section{References}

713 Amara, M., Aquilina, D., Henry, I., \& PMP. (2004). Education of young sportspersons (lot 1). 714 Final report. Brussels: European Commission.

715 Aquilina, D. (2013). A study of the relationship between elite athletes' educational development 716 and sporting performance. The International Journal of the History of Sport, 30, 374-392. doi:10.1080/09523367.2013.765723

718 Aquilina, D., \& Henry, I. (2010). Elite athletes and university education in Europe: A review of 719 policy and practice in higher education in the European Union Member States. International Journal of Sport Policy and Politics, 2, 25-47. doi:10.1080 /19406941003634024

721 Baron-Thiene, A., \& Alfermann, D. (2015). Personal characteristics as predictors for dual career dropout versus continuation: a prospective study of adolescent athletes from German elite sport schools. Psychology of Sport and Exercise, 21, 42-49. doi:10.1016 /j.psychsport.2015.04.006

725 Borggrefe, C., \& Cachay, K. (2012). "Dual Careers": The structural coupling of elite sport and school exemplified by the German Verbundsysteme. European Journal of Sport and Society, 9, 57-80. doi:10.1080/16138171.2012.11687889

Brewer, B. W., Petitpas, A. J., \& Van Raalte, J. L. (2017). Self-identity and young athletes. In C.J. Knight, C.G. Harwood, \& D. Gould (Eds.), Sport psychology for young athletes (pp. 153-163). Abingdon, Oxon: Routledge.

731 Bronfenbrenner, U. (2005). Making human beings human: Bioecological perspectives on human development. Thousand Oaks, CA: Sage.

733 Brown, D. J., Fletcher, D., Henry, I., Borrie, A., Emmett, J., Buzza, A., \& Wombwell, S. (2015).

734 A British university case study of the transitional experiences of student-athletes. Psychology of Sport and Exercise, 21, 78-90. doi:10.1016/j.psychsport.2015.04.002

736 Brown, C. H., Gould, D., \& Foster, S. (2005). A framework for developing contextual 

intelligence (CI). The Sport Psychologist, 19, 51-62. doi:10.1123/tsp.19.1.51

738 Carless, D., \& Douglas, K. (2009). 'We haven't got a seat on the bus for you' or 'all the seats are 739 mine': narratives and career transition in professional golf. Qualitative Research in Sport and Exercise, 1, 51-66. doi:10.1080/19398440802567949

741 Christensen M. K., \& Sørensen, J. K. (2009). Sport or school? Dreams and dilemmas of talented 742 young Danish football players. European Physical Education Review, 15, 115-133. $743 \quad$ doi: $10.1177 / 1356336 \times 09105214$

744 Debois, N., Ledon, A., \& Wylleman, P. (2015). A lifespan perspective on the dual career of elite 745 male athletes. Psychology of Sport Exercise, 21, 15-26. doi:10.1016 /j.psychsport.2014.07.011

European Commission (2011). Developing the European Dimension in Sport. Brussels:

$748 \quad$ European Commission.

749 EU Expert group. (2012). EU guidelines on dual careers of athletes. Recommended policy 750 actions in support of dual careers in high-performance sport. Approved by the EU Expert 751 Group "Education \& Training in Sport" at its meeting in Poznan on 28 September 2012.

752 Harwood, C.G., \& Knight, C.J. (2015). Parenting in youth sport: A position paper on parenting 753 expertise. Psychology of Sport and Exercise, 16, 24-35. doi:10.1016 $754 \quad$ j.psychsport.2014.03.001

755 Henriksen, K., Stambulova, N., \& Roessler, K. K. (2010a). Successful talent development in 756 track and field: considering the role of the environment. Scandinavian Journal of Medicine 757 \& Science in Sports, 20, 122-132. doi:10.1111/j.1600-0838.2010.01187.x

758 Henriksen, K., Stambulova, N., \& Roessler, K. K. (2010b). Holistic approach to athletic talent 759 development environments: A successful sailing milieu. Psychology of Sport and Exercise, 760 11, 212-222. doi:10.1016/j.psychsport.2009.10.005

761 Henriksen, K., Stambulova, N., \& Roessler, K. K. (2011). Riding the wave of an expert: A 
762

763

764

765

766

767

768

769

770

771

772

773

774

775

776

777

778

779

780

781

782

783

784

785

786

successful talent development environment in Kayaking. The Sport Psychologist, 25, 341362. doi:10.1123/tsp.25.3.341

Henry, I. (2010). Elite athletes and higher education: Lifestyle, 'balance' and the management of sporting and educational performance. Retrieved from http://doc.rero.ch/record/22126/files/2010___Henry.pdf

Knight, C. J., \& Holt, N. L. (2011). Sport participation during childhood and adolescence. In N. L. Holt \& M. Talbot (Eds.), Long term engagement in sport and physical activity: Participation and performance across the lifespan (pp. 9-18). Abingdon, Oxon: Routledge.

Knight, C. J., \& Holt, N. L. (2014). Parenting in youth tennis: Understanding and enhancing children's experiences. Psychology of Sport and Exercise, 15, 155-164. doi:10.1016/j.psychsport.2013.10.010

Larsen, C. H., Alfermann, D., Henriksen, M. K., \& Christensen, M. K. (2013). Successful talent development in soccer: The characteristics of the environment. Sport, Exercise, and Performance Psychology, 2, 190-206. doi:10.1037/a0031958

Lupo, C., Guidotti, F., Goncalves, C. E., Moreira, L., Doupona Topic, M., Bellardini, H., et al., (2015). Motivation towards dual career of European student-athletes. European Journal of Sport Science, 15, 151-160. doi:10.1080/17461391.2014.940557

Martindale, R. J., Collins, D., \& Daubney, J. (2005). Talent development: A guide for practice and research within sport. Quest, 57, 353-375. doi:10.1080/00336297.2005.10491862

McCormack, C., \& Walseth, K. (2013). Combining elite women's soccer and education: Norway and the NCAA. Soccer \& Society, 14, 887-897. doi:10.1080/14660970.2013.843927

Miles, M. B., Huberman, A. M., \& Saldaña, J. (2014). Qualitative data analysis: A methods sourcebook ( $\left.3^{\text {rd }} \mathrm{Ed}\right)$. Thousand Oaks, CA: Sage

Rees, T., \& Hardy, L. (2000). An investigation of the social support experiences of high-level sports performers. The Sport Psychologist, 14, 327-347.doi:10.1123/tsp.14.4.327 
787

788

789

790

791

792

793

794

795

796

797

798

799

800

801

802

803

804

805

806

807

808

809

810

811

Ryan, D. C. (2015). Factors impacting carded athlete's readiness for dual careers. Psychology of Sport and Exercise, 21, 91-97. doi:10.1016/j.psychsport.2015.04.008

Ryan, R. M., \& Deci, E. L. (2000). Overview of self-determination theory: An organismic dialectical perspective. In E. L. Deci \& R. M. Ryan (Eds.), Handbook of self-determination research (pp. 3-33). Rochester, NY: The University of Rochester Press.

Ryba, T. V., Stambulova, N. B., Ronkainen, N. J., Bundgaard, J., \& Selänne, H. (2015). Dual career pathways of transnational athletes. Psychology of Sport and Exercise, 21, 125-134. doi:10.1016/j.psychsport.2014.06.002

Smith, B., \& Caddick, N. (2012). Qualitative methods in sport: A concise overview for guiding social scientific sport research. Asia Pacific Journal of Sport and Social Science, 1, 60-73.

Sparkes, A., \& Smith, B. (2009). Judging the quality of qualitative inquiry: Criteriology and relativism in action. Psychology of Sport and Exercise, 10, 491-497. doi:10.1016 /j.psychsport.2009.02.006

Stambulova, N. B., Engström, C., Franck, A., Linnér, L., \& Lindahl, K. (2015). Searching for an optimal balance: dual career experiences of Swedish adolescent athletes. Psychology of Sport and Exercise, 21, 4-14. doi:10.1016/j.psychsport.2014.08.009

Torregrosa, M., Ramis, Y., Pallarés, F., \& Selva, C. (2015). Olympic athletes back to retirement: A qualitative longitudinal study. Psychology of Sport and Exercise, 21, 50-56. doi:10.1016 /j.psychsport.2015.03.003

Wylleman, P., \& Lavallee, D. (2004). A developmental perspective on transitions faced by athletes. In M. Weiss (Ed.), Developmental sport psychology: A lifespan perspective. Morgantown, WV: Fitness Information Technology.

Wylleman, P., Reints, A., \& De Knop, P. (2013). A developmental and holistic perspective on athletic career development. In P. Sotiaradou, \& V. De Bosscher (Eds.), Managing high performance sport (pp. 159-182). New York, NY: Routledge. 
Table 1

Stage 1 Participant Details

\begin{tabular}{lll}
\hline \multicolumn{1}{c}{ Setting } & \multicolumn{1}{c}{ Athlete One } & Athlete 2 \\
\hline Tennis & $\begin{array}{l}\text { Dillon }{ }^{6} \text { is a 15-year-old male international } \\
\text { academy } \\
\text { tennis player. Dillon has frequently } \\
\text { represented Great Britain at competitions }\end{array}$ & $\begin{array}{l}\text { tennis player. Niamh is currently transitioning } \\
\text { from junior to senior competitions. Niamh has }\end{array}$ \\
& $\begin{array}{l}\text { around the world. Dillon has been at the } \\
\text { academy for two years and has been }\end{array}$ & Niamh has recently completed her GCSE \\
& $\begin{array}{l}\text { completing two GCSE qualifications a } \\
\text { year. He is currently completing further }\end{array}$ & BTEC and an A' level course.
\end{tabular}
GCSE qualifications and a BTEC. $\begin{array}{lll}\text { performance } & \text { swimmer, who has represented her country swimmer, who has represented her country in } \\ \text { swimming for the last two years. Lisa is currently a number of national and international }\end{array}$ $\begin{array}{ll}\text { swimming for the last two years. Lisa is currently a number of national and international } \\ \text { squad } & \text { completing her GCSE qualifications at a competitions. Hannah is currently completing }\end{array}$ traditional school. Lisa will continue her A level exams, having finished her AS education into higher education to start her levels in the previous year. She is striving to A level exams next year.

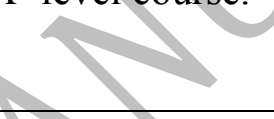
achieve the highest grades in her A' levels. qualifications and is currenty enrolled in a BTEC and an A' level course.

Niamh is a 17 -year-old female international

Social Support Network Members Interviewed

Dillon's Mother

$$
\text { Dillon's Father }
$$

Dillon's coach/academy manager
Niamh's mother
Niamh's coach
$\mathrm{S} \& \mathrm{C}$ coach

Tutor

Performance lifestyle advisor

Hannah's mother Hannah's father

Hannah's sport psychologist

Performance advisor

Hannah and Lisa's coach

Lisa's head of Physical Education

Lisa's head of year Lisa's father

Lisa's mother

\footnotetext{
${ }^{6}$ To protect the identity of the athletes, pseudonyms are used throughout this manuscript
} 


\begin{tabular}{ll}
\hline National & Sofia is a 15-year-old international field \\
field hockey & hockey player. She has competed for her \\
squad & country in a number of international age \\
& group events and is a regular member of the \\
& under 16 and 18 national squad. She has \\
& been part of the national squad for the last \\
& two. Her father is also her coach. Sofia is \\
& currently studying for her GCSE \\
& qualifications within a traditional education \\
& system.
\end{tabular}

National Sofia is a 15-year-old international field

field hockey hockey player. She has competed for her group events and is a regular member of the under 16 and 18 national squad. She has 作 currently studying for her GCSE system.
Cally is a 16-year-old international field hockey player. She has competed for her country in a number of international age group events and is a regular member of the under 18 national squad and also the senior squad. Cally is currently studying for her A level qualifications within a traditional education system. Her club training takes place in the evenings around her schooling with national training occurring monthly.

$$
4
$$

\begin{tabular}{llll}
\hline A football & Brody is a 16-year-old academy football Harry is an 18-year-old academy football & Head of welfare and education \\
academy & player. He has been playing with the player. He attends the academy full-time & Sport psychology consultant \\
Tom's coach & \\
academy for six years. He is currently having completed his GCSE exams at a & Tom's school head of year \\
completing his GCSE exams at a local traditional school almost two years ago. He & Tom's father \\
secondary school. He attends the academy completes 12 hours of education at the & Harry's coach \\
for two afternoons a week (on day release) academy and will obtain a BTEC qualification & Harry's father \\
and trains three evenings a week/plays at the end of this year. He trains every day & \\
games at the weekend. He has an except for Wednesdays and plays matches at \\
opportunity to attend educational sessions the weekend. \\
at the academy to make up the work he \\
misses.
\end{tabular}

Sofia's father

National coach

Sofia's mother

Sofia's form tutor

Cally's father

Cally's mother

Cally's PE teacher

National age group team manager

National team sport psychologist

Head of welfare and education sychology consultant

school head of

Harry's coach

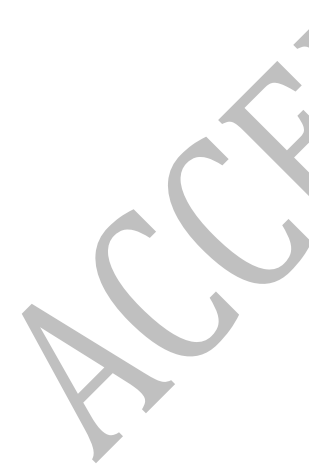


Table 2

Participant demographics

\begin{tabular}{|c|c|c|c|c|c|}
\hline Participants & Number & Gender & Age (years) & Highest Education & Other \\
\hline$\overline{\text { Athletes }}$ & $\overline{8}$ & $\begin{array}{l}\overline{5 \text { female }} \\
3 \text { male }\end{array}$ & $\begin{array}{l}15-18 \\
(M=16.1)\end{array}$ & $\begin{array}{l}4 \text { completing GCSES } \\
4 \text { completing A'levels/BTEC }\end{array}$ & $\begin{array}{l}6 \text { in dual parent family } \\
2 \text { single parent family }\end{array}$ \\
\hline Parents & 13 & $\begin{array}{l}6 \text { female } \\
7 \text { male }\end{array}$ & $\begin{array}{l}39-67 \\
(M=49.9)\end{array}$ & $\begin{array}{l}3 \text { Masters degree } \\
2 \text { graduate diploma } \\
4 \text { undergraduate degrees } \\
2 \text { vocational training/diploma } \\
2 \text { high school leaving qualifications }\end{array}$ & \\
\hline $\begin{array}{l}\text { Coaches/ } \\
\text { Team managers }\end{array}$ & $7^{*}$ & 7 male & $\begin{array}{l}26-56 \\
(M=38.4)\end{array}$ & 7 undergraduate degrees & \\
\hline Teachers/Tutors & 6 & $\begin{array}{l}1 \text { female } \\
5 \text { male }\end{array}$ & $\begin{array}{l}25-37 \\
(M=31.7)\end{array}$ & uate degrees & \\
\hline $\begin{array}{l}\text { Sport scientists } \\
\text { /advisors }\end{array}$ & 7 & $\begin{array}{l}2 \text { female } \\
5 \text { male }\end{array}$ & & graduate degree & $\begin{array}{l}3 \text { sport psychologists } \\
2 \text { performance lifestyle advisors } \\
1 \text { education and welfare officer } \\
1 \text { performance advisor }\end{array}$ \\
\hline
\end{tabular}

*one coach was also a parent 